\title{
Advanced Driver Security Application
}

\author{
Suhas N Bhargav, Rajani Katiyar
}

\begin{abstract}
The introduction of modern and more advanced vehicles has stretched their performance boundaries dramatically in terms of pace and maneuverability. It has also significantly enhanced the likelihood of people losing control of their vehicle, contributing to accidents. Within the past, several strategies have been suggested which resolve this issue by restricting the car's travel to just one specific path. To various road situations, this is achieved by applying lane identification utilizing algorithms such as canny edge identification, Hough transformations, vanishing point estimates, principal component analysis etc. Practical deployment of these programs, however, requires extremely powerful hardware such as TDA3X which can process in real time. This paper aims to introduce the usage of the Lane Detection and Alert System on a Texas Instruments Driver Assist 3x (TDA3x) board with a frame resolution of $(1920 * 1080 \mathrm{p})$ at $2 \mathrm{GHz}$ relative to the current implementation, which has a resolution of only (480 * 270p) at $100 \mathrm{MHz}$ [16]. This system too makes use of canny edge detection and hough transforms to identify the lane points, and tracks the vehicle movement by extracting the corresponding polar co-ordinates.
\end{abstract}

Keywords : Lane Detection and Warning System (LDWS), Canny edge identification, Hough transforms, Vanishing point estimates, Principal component analysis, TDA3x

\section{INTRODUCTION}

$\mathrm{T}$ here could be different causes for an on-road collision. Nonetheless, the split-second pause on the driver's part in reacting to an incident is what determines the condition between life and death. In most instances, accidents result when the driver has little time to react to an impending accident. This is attributed to the loss of synchronization between a driver's body and mind. There are also situations under which the crash is triggered by pure incompetence on the part of the vehicle driver. Whatever could be the cause, a careful rider's timely reaction is only one remedy that may potentially avert a crash and in effect save the lives of others.

Because humans are likely to commit mistakes intentionally or unknowingly, they cannot be expected to assume complete responsibility for maintaining fellow travelers' safety. Within the car an automated device will be built and introduced that can operate independently of the

Revised Manuscript Received on April 25, 2020.

* Correspondence Author

Suhas N Bhargav*, department of Electronics and Communication Engineering, R V College of Engineering, Bengaluru, India. Email: suhas96bhargav@gmail.com

Mrs. Rajani Katiyar, department of Electronics and Communication Engineering, R V College of Engineering, Bengaluru, India. Email: rajanikatiyar@rvce.edu.in

(C) The Authors. Published by Blue Eyes Intelligence Engineering and Sciences Publication (BEIESP). This is an open access article under the CC BY-NC-ND license (http://creativecommons.org/licenses/by-nc-nd/4.0/) human driving the automobile. The purpose of this program is to control the driver's behavior constantly whilst maintaining track of the ambient environments at the same time. The benefit of putting up these devices inside the vehicle is that they can detect the driver's mistakes and anticipate future crashes as well. Not only does this warn the driver of his errors, but it would also help him resolve such errors by taking corrective steps on time.

In this article, Lane Detection and Warning System (LDWS) usecase would be addressed among the other uses that the autonomous systems will have. As the name itself indicates, this program essentially collects video frames from the camera on the front of the vehicle, and then extracts details about the lane lines on the route. The progress of the car will be monitored on the basis of the available details and an appropriate alert will be given to the driver beforehand. Not only does this warn the driver, but it will also help to prevent potential crash until it is too late for the driver to react.

Lane marks were identified on the path by evaluating the double edges of the lane markings [1]. On the captured image, canny edge detection operation was conducted to collect the details about the edges of the road. Then the essential features of the lane markers were derived by making use of this lane edge information and the lane field details. In the other side, [2] sought to concentrate the research in increasing the processor speed rather than on the precision of the usecase. Throughout this method, it was discovered that the usecase processing speed could be significantly improved by the resolution of the captured image, without any loss in precision or efficiency. [3] and [4] concentrated on the noise dimension of the picture by seeking to increase the efficiency and adaptability of the illumination to adjust to various lighting conditions on the route. However, there were drawbacks to their function in the presence of motorcyclists and pedestrians. [5] attempted to demonstrate the particular bottlenecks found in current procedures, such as obstructions created by the movement of cars, ambiguous lane markers, shadow obstructions attributable to houses, etc. and attempted to include several workarounds. [6] and [7] also based their research on solving the question of low road visibility in hilly regions due to the prevalent complex weather conditions including heat, fog, haze, etc. While they were able to obtain an output of $82 \%$ and $92 \%$ respectively, their job had some drawbacks near to the tight curves or in the midst of pedestrians. In [8], a binarization method was applied to the research regions, where each sample area was further divided into two parts: the foreground region and the context zone. This was achieved to make sure the context data did not impact the algorithm's overall output. 
[9] suggested a vision-based lane recognition algorithm to monitor the lanes while less lane markers were present on the route. The algorithm tried to map the lane in this method by utilizing the lane markers from just one side while the other half of the lane markings are totally erased. Also, in cases where both sides of the lane markers were removed, the algorithm sought to allow use of the lane markings from the previous moments and determined the lane points at the present time. Several other works [10] - [15] have sought to conduct vision-based lane recognition, utilizing methods such as perspective analysis, deep neural networks, vanishing point predictions and other mathematical models. Such techniques demanded huge computational costs and high computing capabilities were needed for their implementation.

All of these current works have attempted to conduct lane identification by concentrating on the specific dimension of the problem and have therefore progressed in gaining descent precision. Nonetheless, in order to incorporate these computationally complicated algorithms, ensuring that the execution time is held as short as possible is essential. These kinds of applications often include the use of highly engineered hardware which will allow the captured images to be processed in real time. Texas Instruments has created a hardware named "TDA3x" whose whole function is to support the ADAS framework implementation. This paper thus aims to shed some light on the board and discusses its usage in applying the LDWS usecase.

\section{THEORY AND METHODOLOGY}

As mentioned earlier, software would only be able to run in real time if the equipment it requires is adequately powerful to handle high-speed processing. Consequently, this paper utilizes the TDA3x hardware to execute the usecase addressed. Fig 1 depicts the interfacing diagram for the usecase.

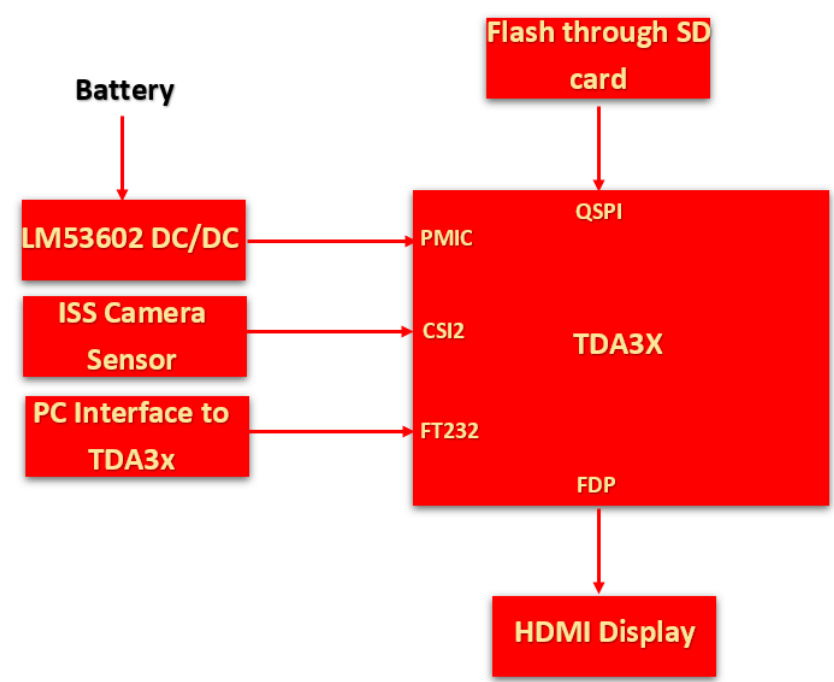

Fig. 1. Use case block diagram of LDWS (This figure indicates the interfaces that needs to be linked to the external $\mathbf{I} / O$ devices for the proper implementation of the proposed system )

There should be a way to import the program code onto the appropriate hardware for deployment in every usecase. This can be achieved by flashing the code via a Secure Display Card (SD card). TDA3x board also takes up a $12 \mathrm{~V}$ supply to get powered up. Because the car's battery can generate a $12 \mathrm{~V}$ voltage drop across its terminal, it is easy to set up this usecase inside the car.

The Fig 2 depicts the methodology used in this paper. If a camera records a video stream, it should be transformed to a gray scale file, because canny edge detection can only operate on monochrome videos. Here, ROI boundary extraction helps to restrict the algorithm's search field and thereby removes any unnecessary information from the pictures collected.

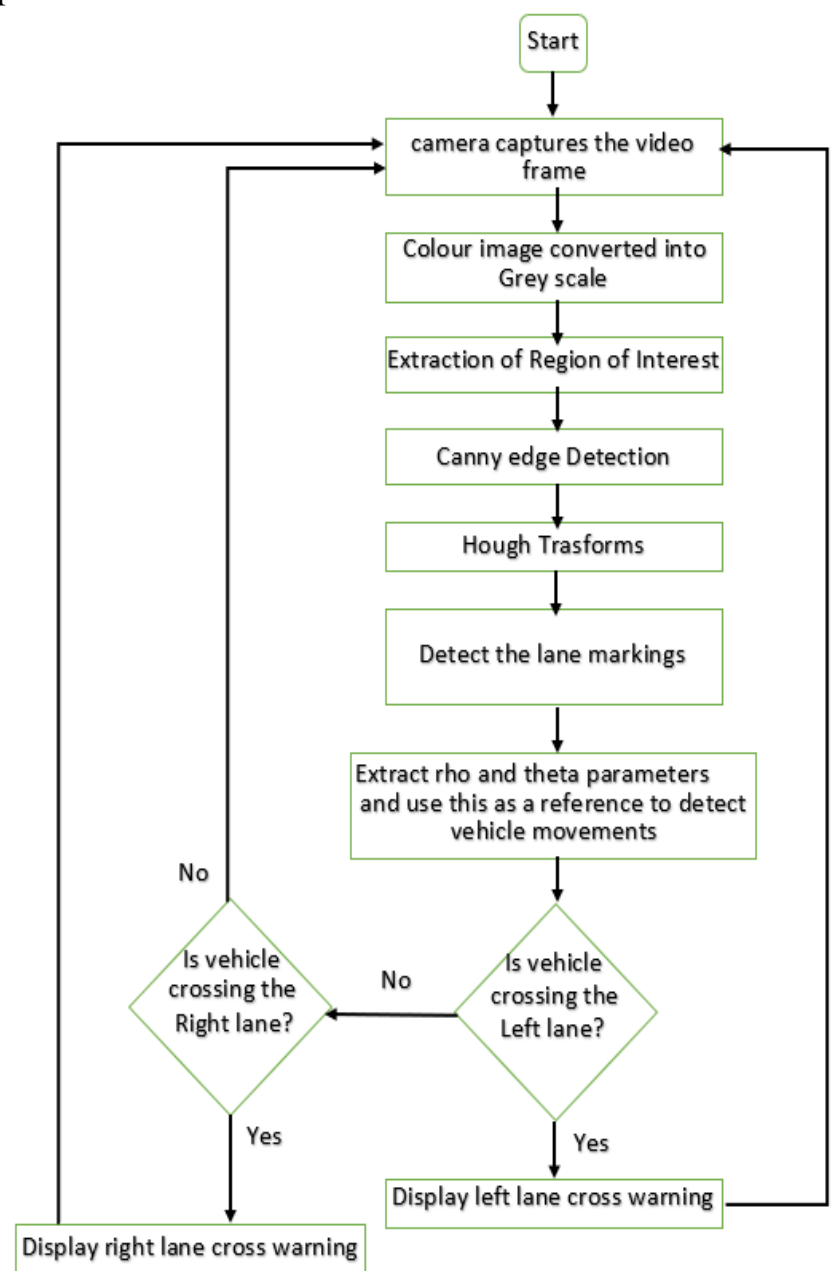

Fig. 2. Flowchart for use in LDWS (This figure depicts the sequence of operations that needs to be performed in the proposed usecase )

While canny edge detection helps to define the edges of the lane in the input picture, Hough transforms can help to establish a mathematical relationship between certain edge points, whether it is a straight line, a parabola or a circle. Hough transforms can also serve to derive the polar equivalents of the lane coordinates acquired to better map the direction of the lane and the test vehicle.

The government agencies who mark the lanes on the road must ensure they travel parallel to each other at all times. Yet in fact the way a camera looks at these lanes would be very special. When the camera is mounted parallel to the ground, it appears that the lines in front of the vehicle behave like a trapezoid, rather than rectangle. The Fig 3 represents this situation.

Published By:

Blue Eyes Intelligence Engineering \& Sciences Publication 
The four extreme vertices of this trapezoid need to be defined first for usecase to classify the trapezoid. It is achieved by extracting the lane points details at the block's Hough Transforms output. The Fig 4 demonstrates the pictorial image of the trapezoid.

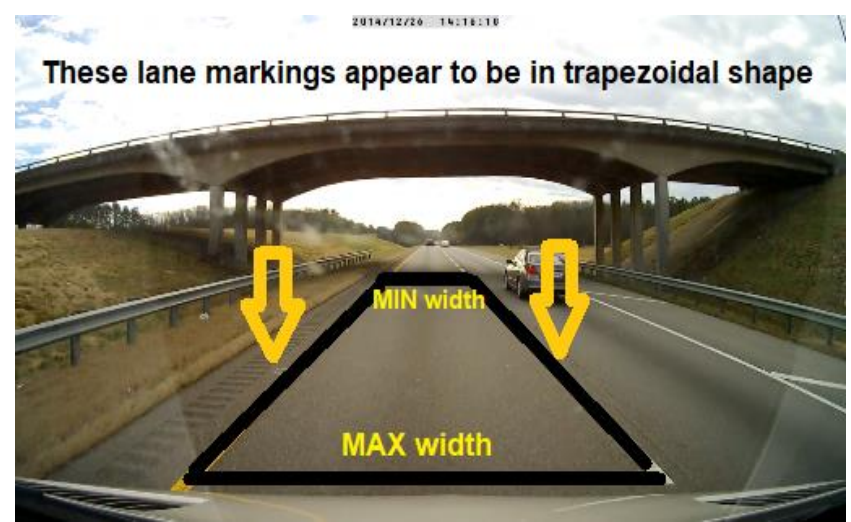

Fig. 3. The camera's viewpoint (This figure presents the practical scenario of the road when viewed by the camera placed in the front of the car)

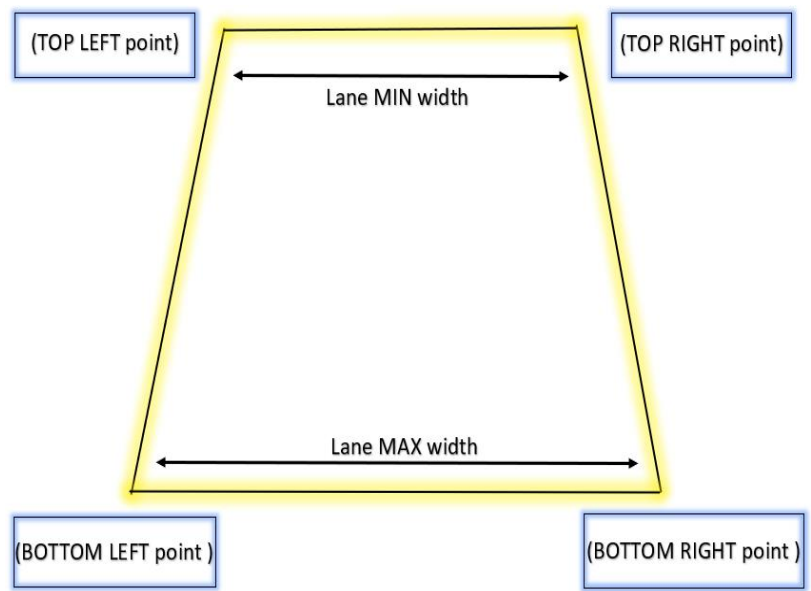

Fig. 4. Pictorial image of the trapezoid (This figure elaborates the consequences of the lane appearing as a trapezoid from the perspective of the camera)

When the 4 vertexes have been obtained, 2-point method can be used to produce a line equation and define the lane markings in effect. Suppose there are two points in space, $(\mathrm{x} 1, \mathrm{y} 1)$ and $(\mathrm{x} 2, \mathrm{y} 2)$. Therefore, the two-point formula for the line connecting those points can be supplied as shown in the equation (2):

$y-y_{1}=\left(\frac{y_{2}-y_{1}}{x_{2}-x_{1}}\right) *\left(x-x_{1}\right)$

where, $\left(\frac{y_{2}-y_{1}}{x_{2}-x_{1}}\right)$ denotes the slope of the line.

The figure above, when generalized, represents the function of the related lane markings. This method can be used to further approximate or forecast the direction of the lane by substituting various values for $\mathrm{x}$ or $\mathrm{y}$ in the method above.

A perpendicular line needs to be constructed from the root to the line to separate the polar parallels (rho and theta) from the specified straight line, as shown in the Fig 5

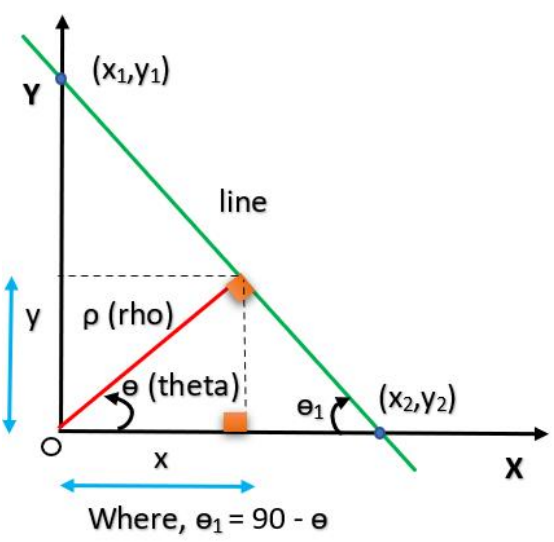

Fig. 5. Extraction of the polar equivalents (This figure depicts the line along the rectangular space and describes how the polar counterparts can be derived mathematically)

Then, using the trigonometric relationship between the angle and the duration of the side, the following equation can be obtained as shown in equation (2):

$\rho=(x * \cos (\theta))+(y * \sin (\theta))$

The definition mentioned above, when applied to both the lanes, results in Fig 6. There, " $\theta_{1}$ " corresponds to the angle subtended by the left lane and " $\theta_{2}$ " corresponds to the angle subtended by the right lane. Likewise, the magnitude of the perpendicular to the left lane is denoted by " $\rho 1$," and the magnitude of the perpendicular to the right lane is denoted by " $\rho 2$ ". The car is said to be in normal condition when it stays within the limits of the current lane.

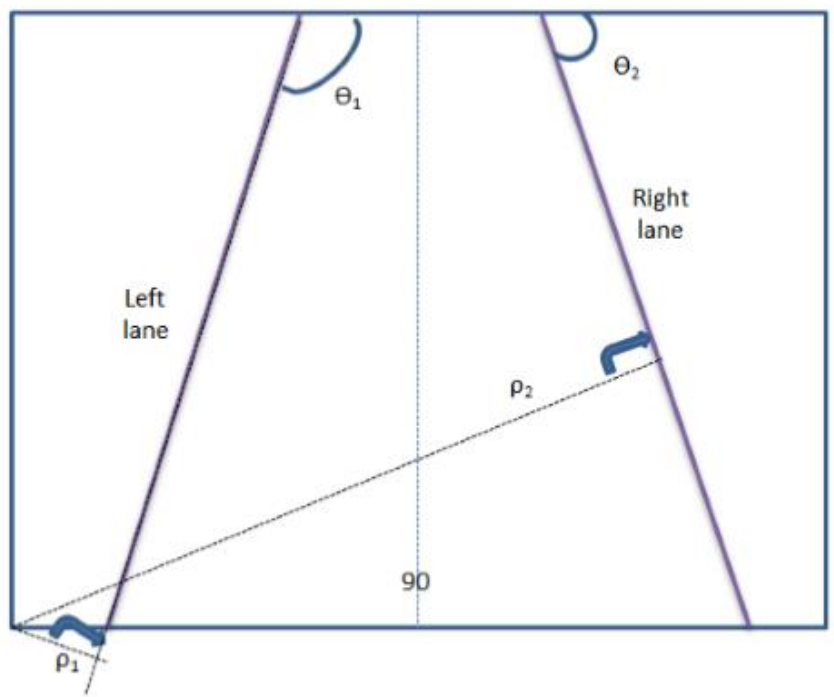

Fig. 6. Image depiction of the lane in normal condition (This figure tries to extend the concept detailed earlier, to practical road conditions)

As the vehicle drifts to the left, the angles undercut by both lanes will decrease, while the magnitude of the perpendicular to the left lane rises and the magnitude of the perpendicular to the right lane drops, as shown in the Fig 7. 


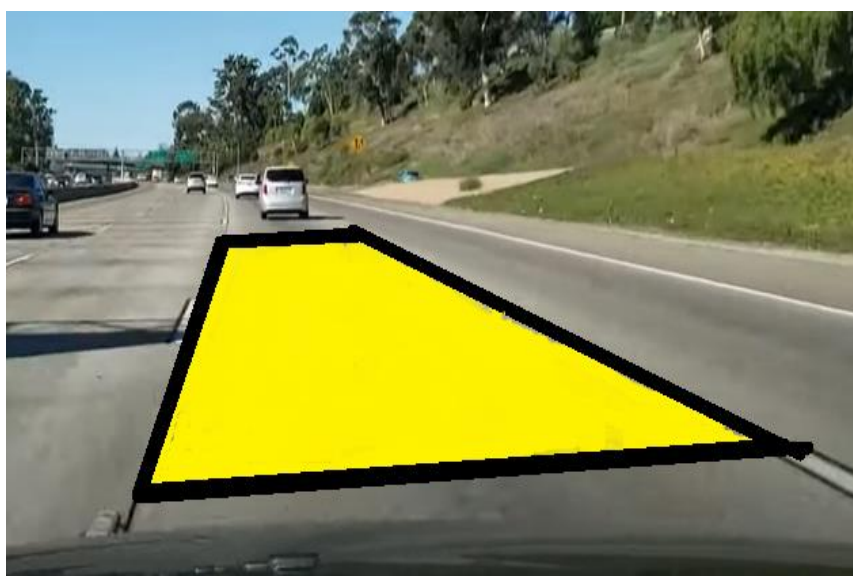

Fig. 7. Image depiction of the lane under left lane drift (This figure helps to visualize how the shape of the lane changes when the vehicle slowly starts moving to the left)

Similarly, as the vehicle drifts to the right, the angles undercut by both lanes will decrease, while the magnitude of the perpendicular to the left lane drops and the magnitude of the perpendicular to the right lane rises, as shown in the Fig 7.

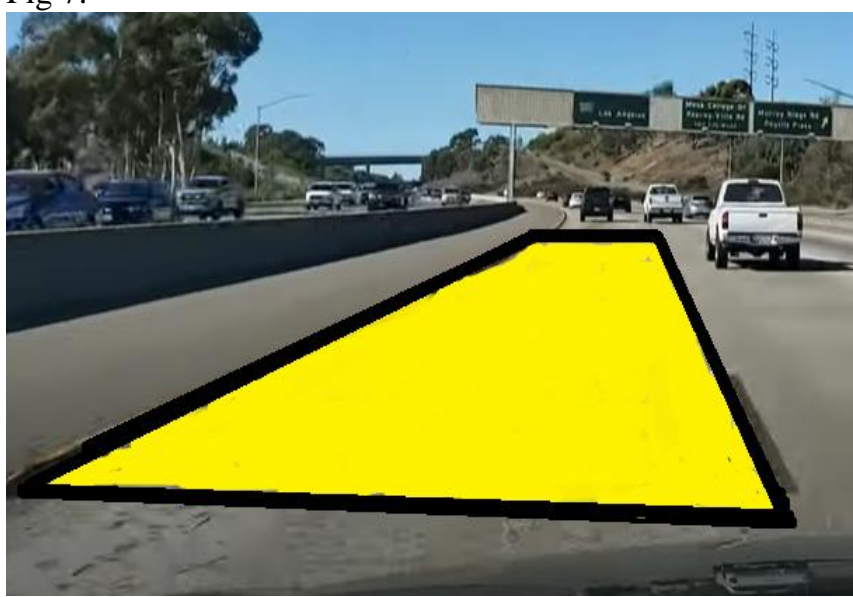

Fig. 8. Image depiction of the lane under right lane drift (This figure helps to visualize how the shape of the lane changes when the vehicle slowly starts moving to the right)

The above principles will be used to get results for the suggested usecase of the LDWS.

\section{RESULT AND DISCUSSION}

Centered on the principles mentioned in the previous section, snapshots of the findings obtained are displayed, accompanied by a thorough review. The Fig 9 displays the snapshot of the lanes observed under normal conditions. The Fig 10 and the Fig 11 shows the snapshots obtained during the lane cross events.

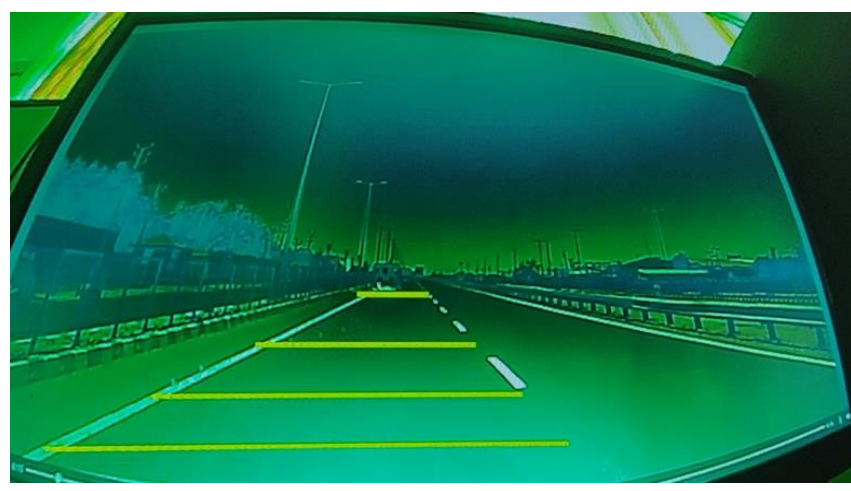

Fig. 9. Snapshot of the estimated lane markings (This figure clearly picturizes the scene, where the proposed usecase estimates the lane markings effectively between the two white coloured edges)

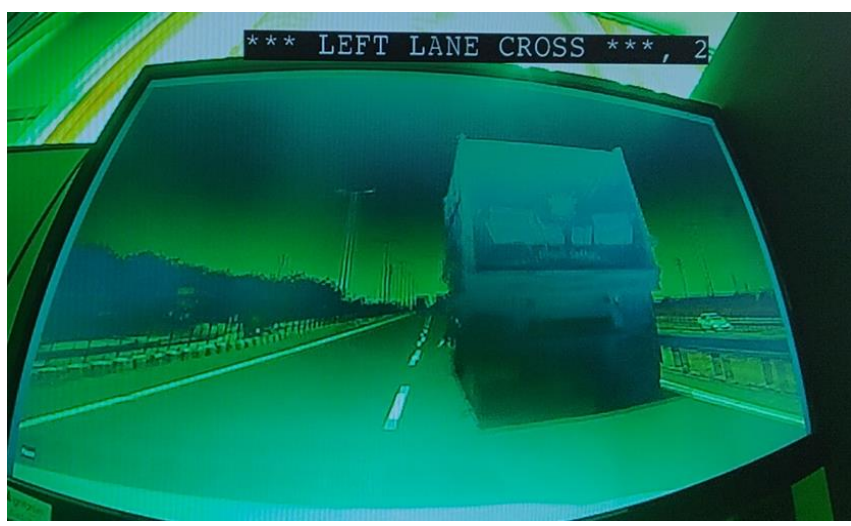

Fig. 10. Snapshot obtained during left lane cross event (This figure was taken at the exact moment when the test vehicle was about to cross the left lane)

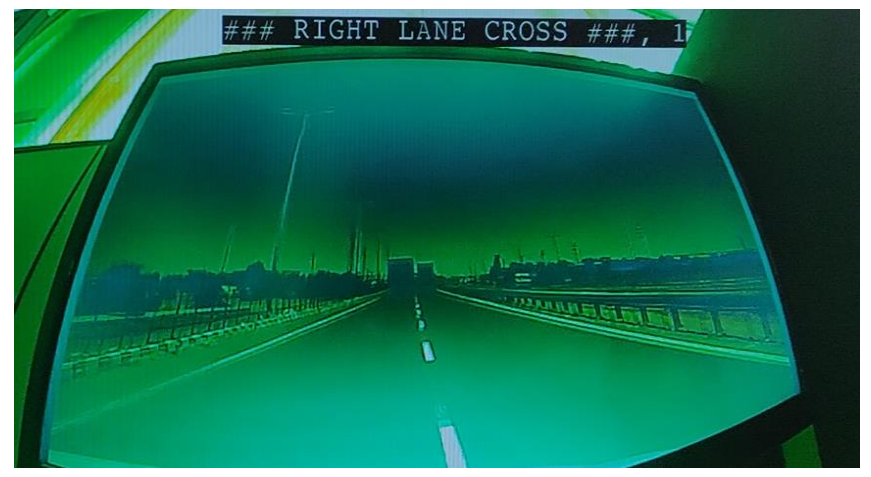

Fig. 11. Snapshot obtained during right lane cross event (This figure was taken at the exact moment when the test vehicle was about to cross the right lane)

The above experiment was also carried out using a sheet of cardboard and a cello tape to imitate the lane lines on the route. The data was evaluated for various camera parameter parameters, and the corresponding readings were tabulated, as seen in table I.

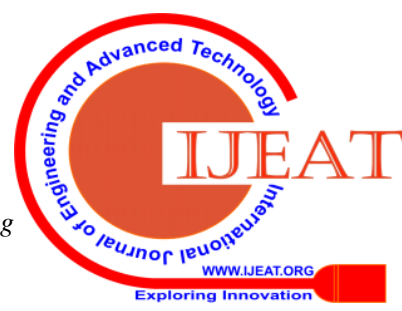


Table- I: Readings for various parameters of the camera

\begin{tabular}{|c|c|c|c|c|c|}
\hline SL. No & $\begin{array}{l}\text { Height of the camera } \\
\text { from ground (inches) }\end{array}$ & $\begin{array}{l}\text { Width of the road } \\
\text { (between the lane } \\
\text { markings) in inches }\end{array}$ & $\begin{array}{c}\text { Camera banking angle } \\
\text { (w.r.t Horizontal axis) in } \\
\text { degrees }\end{array}$ & $\begin{array}{l}\text { Ratio between } \\
\text { Camera height } \\
\text { and road width }\end{array}$ & Result \\
\hline 1 & 3 (least height) & 13.6 & $\begin{array}{c}0 \text { (parallel to the } \\
\text { ground) }\end{array}$ & 0.22 & Lane detected \\
\hline 2 & 3 & 13.6 & $5 \downarrow$ & 0.22 & Lane detected \\
\hline 3 & 3 & 13.6 & $10 \downarrow$ & 0.22 & Lane detected \\
\hline 4 & 3 & 13.6 & $30 \downarrow$ & 0.22 & Lane not detected \\
\hline 5 & 10 & 13.6 & 0 & 0.73 & Lane detected \\
\hline 6 & 10 & 13.6 & $20 \downarrow$ & 0.73 & Lane detected \\
\hline 7 & 15.8 & 13.6 & 0 & 1.161 & Lane not detected \\
\hline 8 & 15.8 & 13.6 & $20 \downarrow$ & 1.161 & Lane detected \\
\hline 9 & 17 & 13.6 & 0 & 1.25 & Lane not detected \\
\hline 10 & 17 & 13.6 & $20 \downarrow$ & 1.25 & Lane detected \\
\hline 11 & 3 & 25.3 & 0 & 0.11 & Lane not detected \\
\hline 12 & 10 & 25.3 & 0 & 0.39 & Lane detected \\
\hline 13 & 10 & 25.3 & $10 \downarrow$ & 0.39 & Lane detected \\
\hline 14 & 10 & 25.3 & $20 \downarrow$ & 0.39 & Lane detected \\
\hline 15 & 17 & 25.3 & 0 & 0.67 & Lane detected \\
\hline 16 & 17 & 25.3 & $20 \downarrow$ & 0.67 & Lane detected \\
\hline 17 & 24.5 & 25.3 & 0 & 0.968 & Lane not detected \\
\hline 18 & 24.5 & 25.3 & $15 \downarrow$ & 0.968 & Lane detected \\
\hline 19 & 30 & 25.3 & 0 & 1.18 & Lane not detected \\
\hline 20 & 30 & 25.3 & $25 \downarrow$ & 1.18 & Lane detected \\
\hline 21 & 35 & 25.3 & $30 \downarrow$ & 1.38 & Lane detected \\
\hline
\end{tabular}

Once the collected readings from the tabular column have been evaluated, it can be inferred that the efficiency of the proposed application depends on the relative difference between camera height and road width. As the ratio of these two values begins to rise, it gets more harder for the usecase to accurately identify the lanes. Based on the same arguments, the lane markers on the path cannot be correctly measured when the ratio gets too small of a magnitude. This is because in both instances, the lanes in the shot picture stretch way outside the algorithm's processing range.

Unless at all the consumer also needs to be able to accurately identify the lanes at higher ratios, the device will be tilted downwards by a slight angle such that it is no longer parallel to the road surface. However, the optimal ratio the code should operate at, though, is in the range 0.3 to 0.8 .

\section{CONCLUSION AND FUTURE SCOPE}

Existing lane detection techniques on the Xilinx Zynq-7000 APSoC platform allow only a resolution of $(480 \times 270$ p $)$ at $100 \mathrm{MHz}$ [16]. This paper tries to implement the proposed usecase by making use of a highly efficient and user-friendly hardware, the TDA3x board that allows maximum HD quality $(1920 \times 1080 \mathrm{p})$ at $2 \mathrm{GHz}$. As a consequence, the lane markers from the recorded video streams will be clearer and the sensed edges will be very precise. Also, the hardware level requirements for powering up this board makes it the most suitable one for setting up inside the vehicles. The availability of multiple cores in the hardware help to solve the computationally complex processes with ease. Overall, this paper is successful in providing a solution for a real-time implementation of the proposed safety application.

However, the proposed approach may be of some value when the driver is completely awake. In situations, when the driver drowses off, there is no significance of these warnings.

Also, these warnings will become a nuisance to the driver when he is deliberately wanting to change the lane. To addresses these minor issues, a more intelligent system can be developed in the future.

\section{ACKNOWLEDGMENT}

The writers would like to thank Mr. Ameermon V A, senior staff Engineer, Ignitarium Technology solutions Private Limited, for his continuous assistance and motivation.

\section{REFERENCES}

1. Ganlu Deng, Yefu Wu, "Double Lane Line Edge Detection Method Based on Constraint Conditions Hough Transform", 2018 17th International Symposium on Distributed Computing and Applications for Business Engineering and Science (DCABES), 19-23 Oct. 2018.

2. Li Dang, Girma Tewolde, Xiaoyuan Zhang, Jaerock Kwon, "Reduced resolution lane detection algorithm”, 2017 IEEE AFRICON, 18-20 Sept. 2017.

3. Mehdi FENICHE, Tomader MAZRI, "Lane Detection and Tracking for Intelligent Vehicles: A Survey", 2019 International Conference of Computer Science and Renewable Energies (ICCSRE), 22-24 July 2019.

4. Alireza Bosaghzadeh, Seidfarbod Seidali Routeh, "A novel PCA perspective mapping for robust lane detection in urban streets", 2017 Artificial Intelligence and Signal Processing Conference AISP), 25-27 Oct. 2017.

Published By:

Blue Eyes Intelligence Engineering \& Sciences Publication

(C) Copyright: All rights reserved. 
5. Suvarna Shirke, C. Rajabhushanam, "A study of lane detection techniques and lane departure system”, 2017 International Conference on Algorithms, Methodology, Models and Applications in Emerging Technologies (ICAMMAET), 16-18 Feb. 2017.

6. Kodeeswari Manoharan, Philemon Daniel, "A Robust Approach for Lane Detection in Challenging Illumination Scenarios", 2018 International Conference on Advances in Computing, Communication Control and Networking (ICACCCN), 12-13 Oct. 2018.

7. Nima Zarbakht, Ju Jia Zou, "Lane Detection under Adverse Conditions Based on Dual Color Space", 2018 Digital Image Computing: Techniques and Applications (DICTA), 10-13 Dec. 2018.

8. Xuqin Yan, Yanqiang Li, "A method of lane edge detection based on Canny algorithm”, 2017 Chinese Automation Congress (CAC), 20-22 Oct. 2017.

9. Yueen Ma, Vincent Havyarimana, Jing Bai, Zhu Xiao, "Vision-Based Lane Detection and Lane-Marking Model Inference: A Three-Step Deep Learning Approach", 2018 9th International Symposium on Parallel Architectures, Algorithms and Programming (PAAP), 26-28 Dec. 2018.

10. Van-Quang Nguyen, Changjun Seo, Heungseob Kim, Kwangsuck Boo, "A study on detection method of vehicle based on lane detection for a driver assistance system using a camera on highway", 2017 11th Asian Control Conference (ASCC), 17-20 Dec. 2017.

11. Huifeng Wang, Yunfei Wang, Xiangmo Zhao, Guiping Wang, He Huang, Jiajia Zhang, "Lane Detection of Curving Road for Structural Highway with Straight-Curve Model on Vision", IEEE Transactions on Vehicular Technology, vol: 68, issue: 6, pp: 5321 - 5330, June 2019.

12. Chung-Bin Wu, Li-Hung Wang, Kuan-Chieh Wang, "Ultra-Low Complexity Block Based Lane Detection and Departure Warning System ", IEEE Transactions on Circuits and Systems for Video Technology, vol: 29 , issue: 2, pp: 582 - 593, Feb. 2019.

13. Yang Xing,Chen Lv, Long Chen, Huaji Wang, Hong Wang, Dongpu Cao, Efstathios Velenis, Fei-Yue Wang, "Advances in Vision-Based Lane Detection: Algorithms, Integration, Assessment, and Perspectives on ACP-Based Parallel Vision", IEEE/CAA Journal of Automatica Sinica, vol: 5, issue: 3, pp: 645 - 661, May 2018.

14. Chanho Lee, Ji-Hyun Moon, "Robust Lane Detection and Tracking for Real-Time Applications", IEEE Transactions on Intelligent Transportation Systems, vol: 19, issue: 12, pp: 4043 - 4048, Dec 2018.

15. Yingna Su, Yigong Zhang, Tao Lu, Jian Yang, Hui Kong, "Vanishing Point Constrained Lane Detection with a Stereo Camera", IEEE Transactions on Intelligent Transportation Systems, vol: 19, issue: 8, pp: 2739 - 2744, Aug. 2018.

16. Chanon Khongprasongsiri, Pinit Kumhom, Watcharapan Suwansantisuk, Teerasak Chotikawanid, Surachate Chumpol, Masami Ikura , "A Hardware Implementation for Real-Time Lane Detection using High-Level Synthesis", 2018 International Workshop on Advanced Image Technology (IWAIT), 7-9 Jan. 2018

\section{AUTHORS PROFILE}

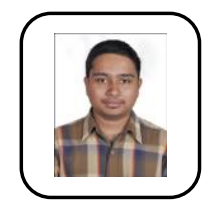

Suhas N Bhargav is a postgrad student doing his masters in the field of Communication systems in Department of Electronics and Communication Engineering, RV college of engineering, Bengaluru. He has obtained Bachelor of Engineering degree in Electronics and Communication Engineering domain from BNM Institute of Technology, Bengaluru in 2018. He has published 2 papers in IEEE International conferences on recent trends in electronics. He has also participated in national level technical exhibitions held in IIT- Delhi. His areas of interest include Communication Systems, Networking, Embedded system design, Image Processing, Digital circuit design, and Signals and Systems.

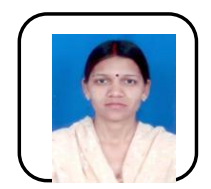

Rajani Katiyar is working as Assistant Professor, Department of Electronics and Communication Engineering, RV college of Engineering, Bengaluru. She has got 14 years of teaching experience. She has obtained Bachelor of technology degree from MJPRU Bareilly in the year 2001 and obtained Master of Technology degree from DAVV University, Indore in 2005. She completed her PhD degree in the year 2020 from Visveswaraya Technological University, Belagavi, India. She has published 1 paper in National conferences, 3 papers in IEEE International conferences and 5 papers in International journals. Her area of interests includes Broadband Communication and Signal Processing.

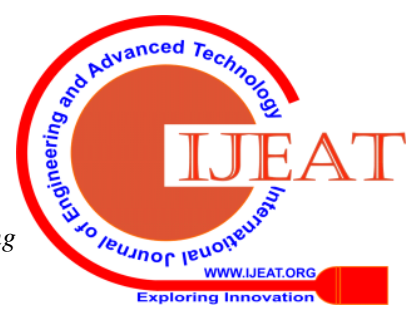

